\title{
The Contrasting Role of the Mediator Subunit MED30 in the Progression of Bladder Cancer
}

\author{
ISABELLA SYRING ${ }^{1}$, RICHARD WEITEN ${ }^{1}$, TIM MÜLLER ${ }^{2}$, DORIS SCHMIDT ${ }^{1}$, SUSANNE STEINER ${ }^{2}$, \\ GLEN KRISTIANSEN ${ }^{2}$, STEFAN C. MÜLLER ${ }^{1 *}$ and JÖRG ELLINGER ${ }^{1 *}$ \\ ${ }^{1}$ Clinic for Urology and Paediatric Urology, University Hospital of Bonn, Bonn, Germany; \\ ${ }^{2}$ Institute of Pathology, University Hospital of Bonn, Bonn, Germany
}

\begin{abstract}
Background/Aim: The Mediator complex is a key regulator of gene transcription, and several studies have demonstrated altered expression of particular subunits in diverse human diseases, especially cancer. To date, nothing is known about the role of MED30 in bladder cancer. Materials and Methods: We, therefore, performed an RNA expression and survival analysis of the subunit MED30 in 537 samples of bladder cancer by using the database cBioPortal. To validate these data on the protein level, we practiced immunohistochemical staining against MED30 on a tissue microarray containing 210 samples of all tumour stages and performed survival analyses. For functional analysis, the siRNA-mediated knockdown of MED30 was performed in the cell lines T24 and TCCSUP followed by proliferation, migration, and invasion assays. Results: On the MRNA and protein levels, higher expression of MED30 is associated with better patient survival. In accordance with this, advanced $T$ - and $N$-stages showed lower expression of MED30. In contrast, knockdown of MED30 led to reduction of the tumour parameters proliferation, migration, and invasion in the BCa cell lines. Conclusion: MED30 appears to be integrated in the progression of the urothelial tumour in the bladder.
\end{abstract}

In Germany, urinary bladder cancer $(\mathrm{BCa})$ was the fourth and 14th most common malignancy in men and women, respectively, in 2012 (1). Approximately 5,400 people, a quarter of whom were women, were diagnosed with invasive

*These Authors contributed equally to this study.

Correspondence to: Isabella Syring, MD, Clinic for Urology and Paediatric Urology, University Hospital of Bonn, Sigmund-FreudStraße 25, 53127 Bonn, Germany. Tel: +49 22828714184, Fax: +49 22828719150, e-mail: isabella.syring@ukbonn.de

Key Words: Mediator complex, bladder cancer, MED30, tumour suppressor. urinary bladder cancer in 2012. In addition, approximately 13,500 patients suffered from non-invasive papillary carcinomas and in situ tumours of the bladder. Above all, the latter have a high tendency towards progression and recurrence. Urinary bladder cancers consist predominantly of urothelial carcinomas, which frequently occur at the same time in different parts of the bladder and urinary tract (1).

Environmental and genetic factors are significant in the development and progression of bladder cancer, although the mechanisms underlying carcinogenesis remain to be fully elucidated. Thus, identifying potential carcinogenic genes is important to develop novel therapeutic strategies and specify novel biomarkers for the diagnosis and prognosis of bladder cancer $(2,3)$.

MED30 is part of the multi-protein Mediator complex, that is separated into four modules: the head, middle, tail and kinase. The Mediator complex is essential for the transcription of protein-coding genes and serves as a hub for diverse signaling pathways. MED30 is a subunit of the head module. The head and middle modules are known to interact directly with RNA polymerase II, whereas the tail module interacts with gene-specific regulatory proteins (4-7).

To date, the only evidence of a possible implication of this subunit in cancers comes from a study on various breast carcinoma cell lines that express abundant amounts of $M E D 30$ (8). Another study shows overexpression of $M E D 30$ in gastric cancer tissues and cell lines (9). Moreover, MED30 overexpression increased proliferation, migration and invasion of gastric cancer cells, whereas MED30-knockdown inhibited these effects.

In a prior analysis by our working group of The Cancer Genome Atlas (TCGA) Database (unpublished data), MED30 was frequently amplified in diverse solid tumours. In $\mathrm{BCa}$, $M E D 30$ showed an amplification frequency of approximately $20 \%$, whereas no deletion of MED30 was detected.

This evidence and the low-abundance of data regarding the role of MED30 in bladder carcinoma prompted us to investigate the relevance of MED30 in urothelial carcinoma of the bladder. 


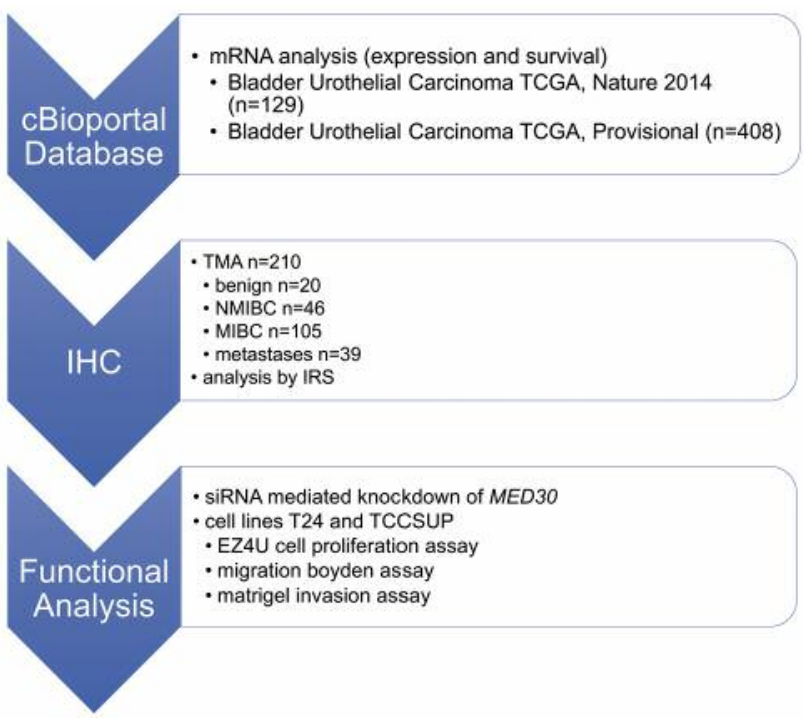

Figure 1. Schema of the analysis-workflow of the study.

\section{Materials and Methods}

Ethics statement. This investigation was conducted in accordance with ethical standards and according to the Declaration of Helsinki and national and international guidelines. Ethical approval for using human material in the present study was obtained from the Internal Review Board of the University Hospital of Bonn (IRB\# 036/08 and \#093/12). The study participants were anonymized before their specimens were included to the study cohort. The entire workflow of the whole study is summarized in Figure 1.

RNA expression analysis by cBioPortal. The database cBioPortal for cancer genomics (http://cbioportal.org, Version 1.4.0) was utilized to investigate the mRNA expression of MED30 in BCa extended by survival analysis. cBioPortal provides a Web resource for exploring, visualizing and analysing multidimensional cancer genomics data. The portal reduces molecular profiling data from cancer tissues and cell lines into readily understandable genetic, epigenetic, gene expression and proteomic events $(10,11)$. For the analysis, RNA sequencing data from "The Cancer Genome Atlas" (TCGA) (12) were used (RNA Seq V2 RSEM) with two SDs from the mean and a threshold \pm 2.0 . The mRNA $z$-scores are precomputed from the expression values and are compared to the expression distribution of each gene tumours that are diploid for this gene [9]. Two mRNA analyses for bladder urothelial carcinoma, named "Bladder Urothelial Carcinoma TCGA, Nature 2014" and "Bladder Urothelial Carcinoma TCGA, Provisional", are available on cBioPortal with 129 and 408 samples, respectively.

Tissue microarray construction. The bladder cohort used in this study, provided by the Clinic for Urology of the University Hospital Bonn, contained 20 benign samples, 46 non-muscle invasive bladder cancer (NMIBC) samples, 105 muscle invasive bladder cancer (MIBC) samples and 39 metastases, in total $n=210$ samples (Table I).
Table I. Clinical pathological data of the bladder cohort.

\begin{tabular}{lccc}
\hline & $\begin{array}{c}\text { BCa } \\
\Sigma=151(\%)\end{array}$ & $\begin{array}{c}\text { Metastasis } \\
\Sigma=39(\%)\end{array}$ & $\begin{array}{c}\text { Benign } \\
\Sigma=20(\%)\end{array}$ \\
\hline Gender & & & \\
Male & $114(75.5)$ & $11(28.2)$ & $13(65.0)$ \\
Female & $37(24.5)$ & $2(5.1)$ & $7(35.0)$ \\
Age & & & \\
Mean & 66.96 & 65.22 & 65.61 \\
Median & 69.00 & 66.00 & 65.00 \\
Range & $36-94$ & $38-84$ & $43-81$ \\
TNM & & & - \\
Tis & $16(10.6)$ & - & - \\
T1 & $30(19.9)$ & - & - \\
T2 & $40(26.5)$ & - & - \\
T3 & $33(21.8)$ & - & - \\
T4 & $32(21.2)$ & - & - \\
N+ & $36(23.8)$ & - & - \\
M+ & $4(2.6)$ & - & - \\
Cancer-associated death & $38(25.2)$ & & - \\
Relapse & $79(52.3)$ & & - \\
Grading & & & - \\
G1 & $3(2.0)$ & - & - \\
G2 & $55(36.4)$ & - & - \\
G3 & $93(61.6)$ & - & - \\
NMIBC & $46(30.5)$ & - & - \\
MIBC & $105(69.5)$ & - & - \\
\hline
\end{tabular}

NMIBC, Non-muscle invasive bladder cancer; MIBC, muscle invasive bladder cancer.

Tissue microarrays (TMA) were performed as described previously $(13,14)$. Briefly, formalin-fixed paraffin-embedded (FFPE) tissues were cut into $4-\mu$ m-thick sections and mounted on slides. After staining with haematoxylin and eosin (H\&E), relevant areas of benign tissue and primary tumour were identified and circled by a pathologist. Each tumour and corresponding benign region was represented with up to three cores measuring $0.6 \mathrm{~mm}$ in diameter on a TMA recipient block using a semiautomatic tissue arrayer (Beecher Instruments, Sun Prairie, WI, USA). H\&E TMA sections were assessed again to confirm the histology.

MED30 protein expression analysis by immunohistochemistry (IHC) in bladder cancer. Prior to performing IHC analyses in the selected TMAs, the specificity of the antibody was confirmed according to the manufacturer's instructions by using stomach cancer as a positive control. IHC was performed using the Ventana Benchmark automated staining system (Ventana Medical System, Tuscon, AZ, USA) as described previously (15-18). Briefly, slides were incubated with the primary antibodies according to the manufacturer's instructions: anti-MED30 rabbit polyclonal (1:250, clone 16787-1-AP, Proteintech, Chicago, IL) at room temperature; antibody dilution was conducted using a Ventana diluent. For signal detection, the ultraView Universal DAB detection kit (Ventana Medical System, Tuscon, AZ, USA) was used. Finally, slides were counter-stained with haematoxylin and bluing reagent, dehydrated and mounted. 


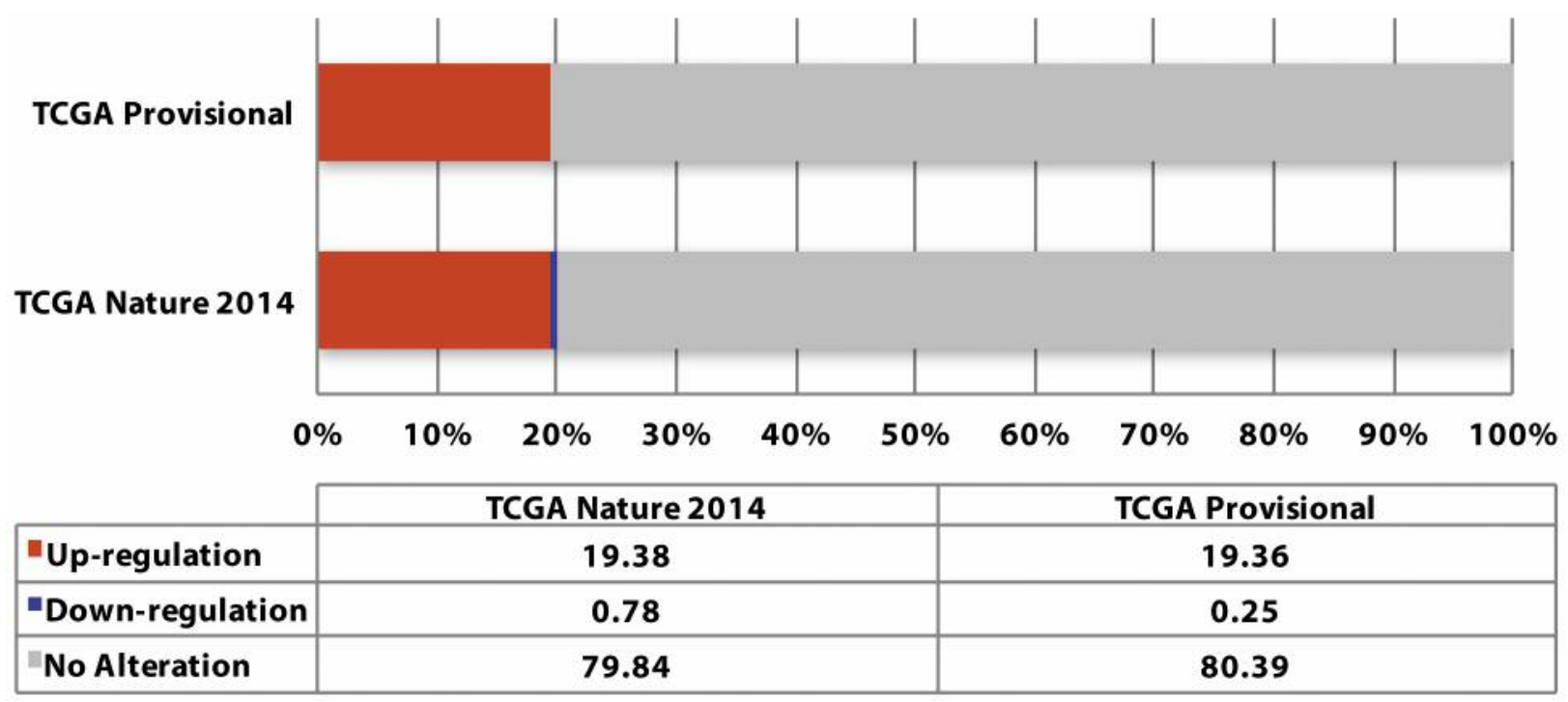

Figure 2. Transcriptional expression analysis of MED30 by cBioPortal in BCa. The mRNA expression [RNA Seq V2 RSEM] of MED30 in bladder urothelial cancer by the database cBioPortal for Cancer Genomics. Two mRNA analyses for bladder urothelial carcinoma, named "Bladder Urothelial Carcinoma TCGA, Provisional" and "Bladder Urothelial Carcinoma TCGA, Nature 2014", are available on cBioPortal with 408 and 129 samples, respectively. The expression is given in \%, divided into "up-regulation", "down-regulation" and "no alteration".

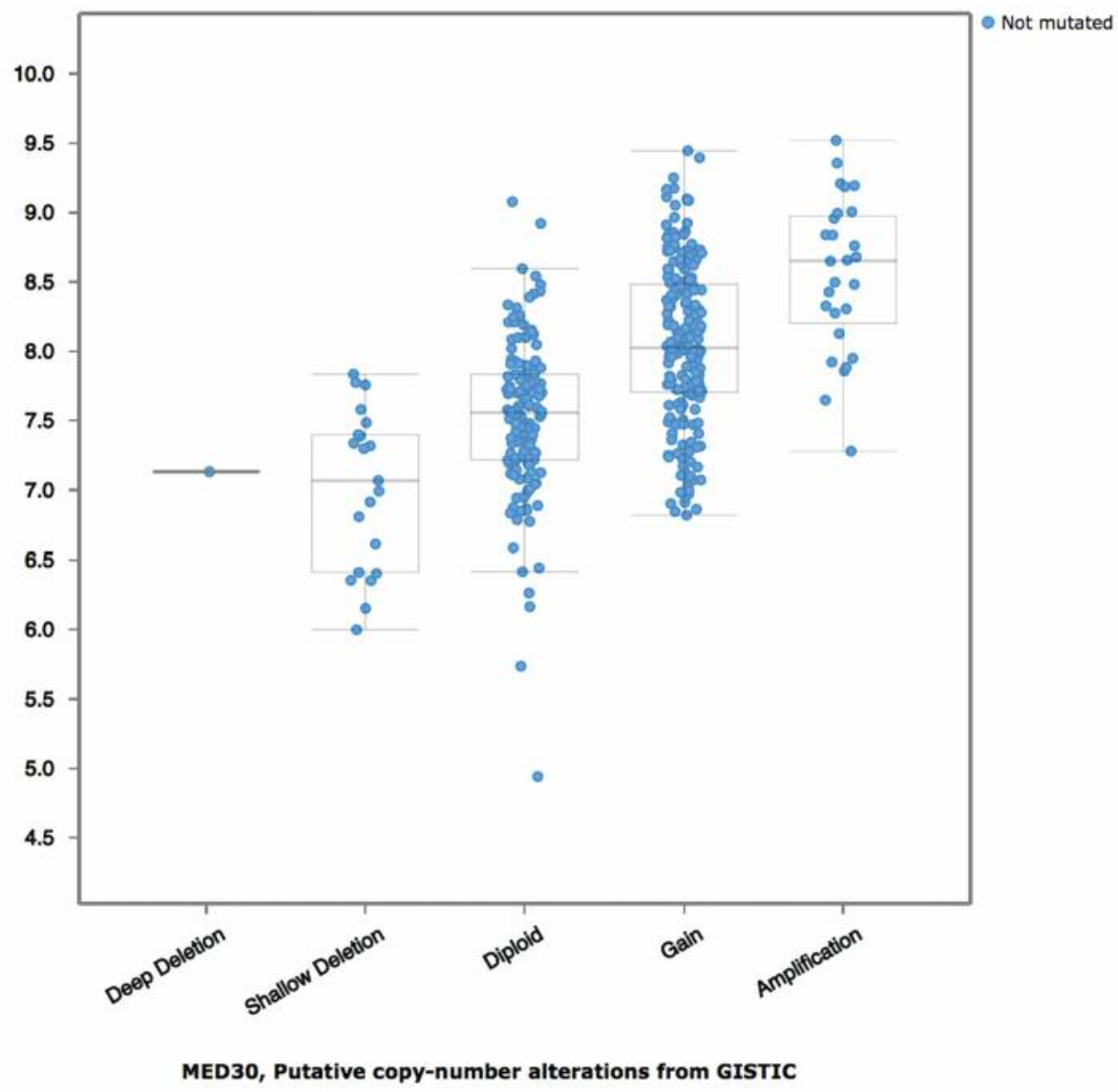

Figure 3. Analysis of the mutation types for MED30 in BCa given by cBioportal. It is shown that the alterations for MED30 in BCa are especially gain-of-function-mutations and amplifications. Deletions are rare. 
TCGA, Provisional
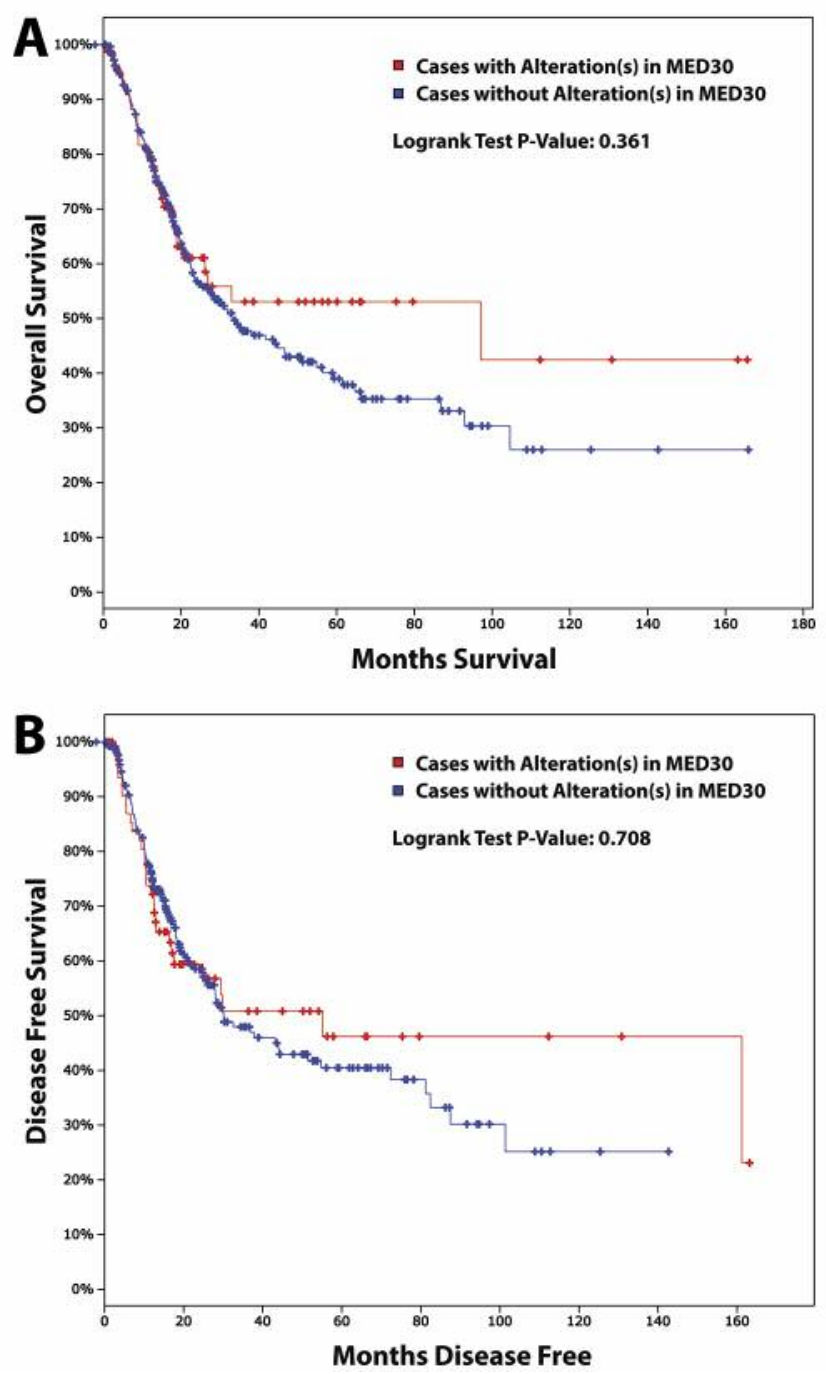

TCGA, Nature 2014
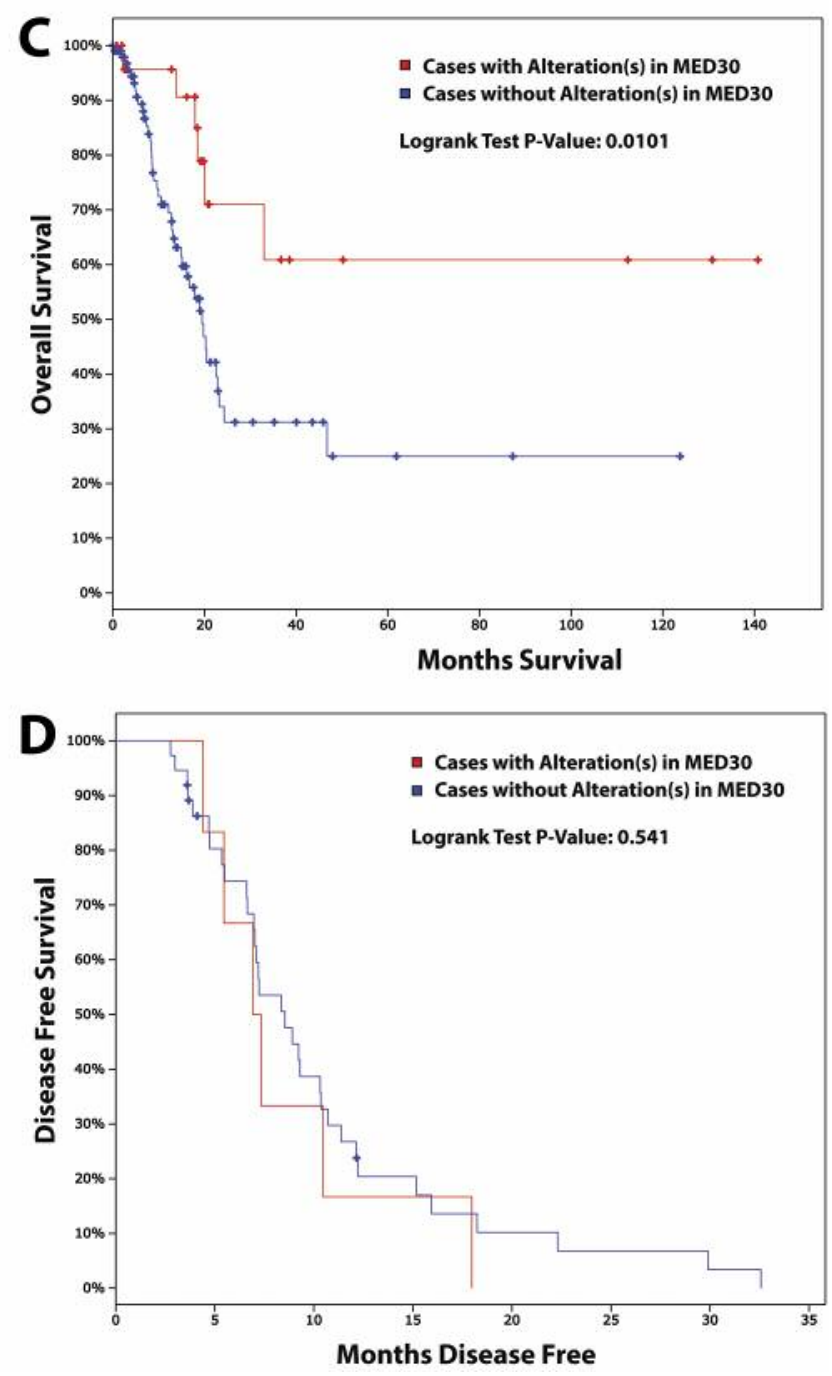

Figure 4. Survival analysis by MED30 mRNA expression on cBioPortal in BCa. Two mRNA analyses for bladder urothelial carcinoma, named "Bladder Urothelial Carcinoma TCGA, Provisional" (A, B) and "Bladder Urothelial Carcinoma TCGA, Nature 2014" (C, D), are available on cBioPortal with 408 and 129 samples, respectively. The overall survival $(A, C)$ and the disease-free survival $(B, D)$ are shown in months by Kaplan-Meier analysis.

After IHC was performed, the staining was evaluated independently by two pathologists (GK, TM). Only cases with at least one assessable TMA core with sufficient tumour tissue were included in the analysis.

Quantification of protein expression was evaluated according to the Immunoreactivity Score (IRS) (19). Briefly, IRS considers the amount of immunoreactive cells as well as the intensity of the nuclear staining. IRS is calculated as the "percentage of immunoreactive tumour cells" $(0=0 \%, 1=\leq 10 \%, 2=10 \%-50 \%, 3=51 \%-80 \%, 4=\geq 80 \%)$ $\times$ "nuclear staining intensity" (from $0=$ no expression to $3=$ strong expression). An IRS of 0 was interpreted as none or a weak expression. An IRS from 1 to 3 was interpreted as a weak to medium expression. An IRS of 4 and above was interpreted as a high expression (overexpression). We decided on a score of 4 or higher, as it reflects cases which either visibly express MED30 in virtually all cancer cells, or harbour at least a marked or strong expression in $11 \%-50 \%$ (or more) of cancer cells. Notably, the statistical correlations were performed with the full-range MED30 IRS and not with the dichotomized categories (i.e. overexpression $v s$. weak-tomedium expression), thus the distinction between overexpressing and non-overexpressing cases had no effect on these analyses.

Clinical data and statistics. Associations with clinico-pathological parameters were performed. Survival analysis was evaluated by Kaplan-Meier estimator and log-rank tests. Statistical evaluation was performed using the Student's $t$-test by Microsoft Excel and SPSS. The data were analysed anonymously. 

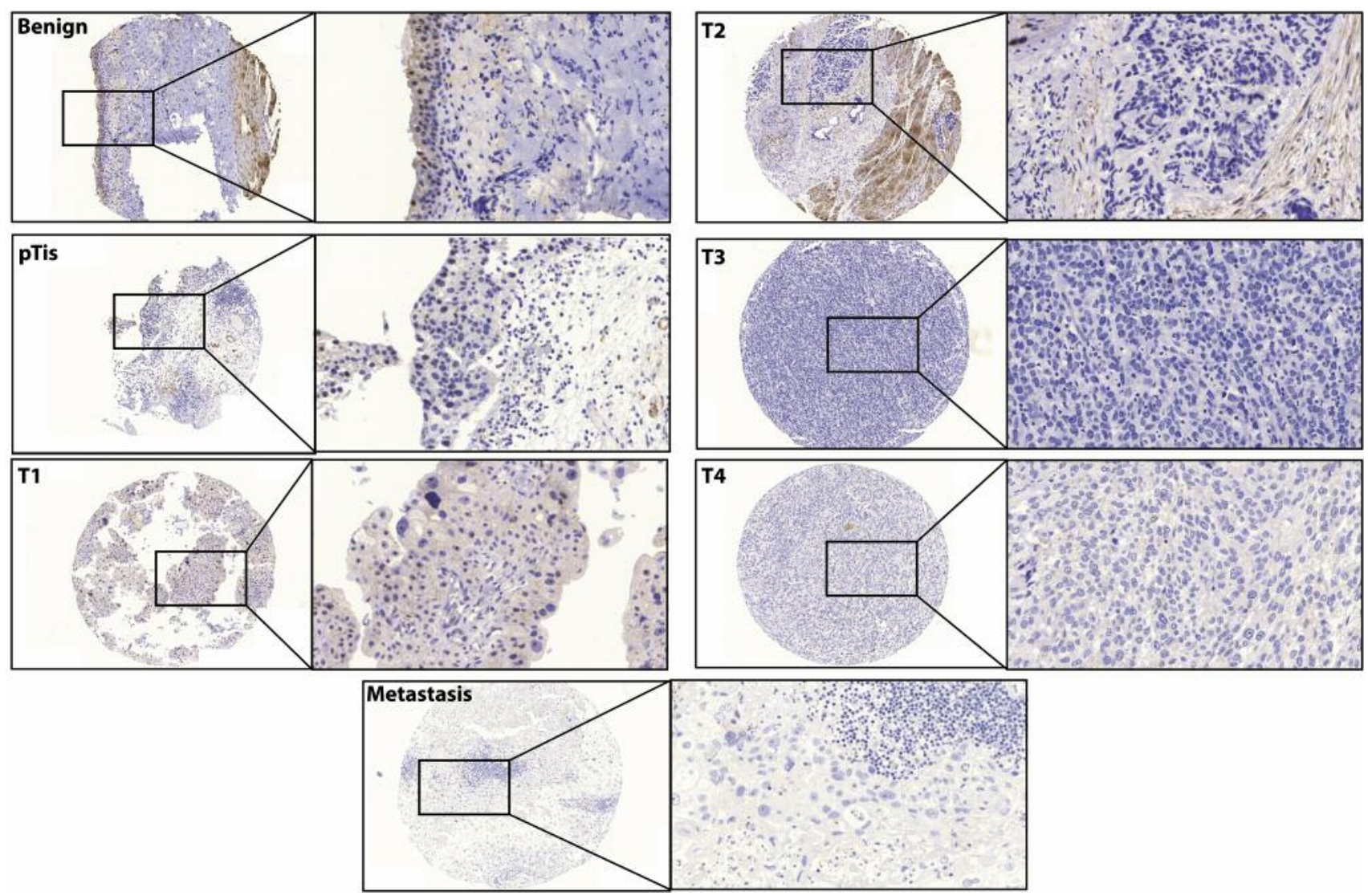

Figure 5. MED30 in BCa by IHC. A. Representative IHC images from tissue of benign urothelium, pTis, T1, T2, T3, T4 and metastatic BCa tissue of the MED30 protein expression. Whole core 10x and inserts 40x objective magnification.

Cell lines and culture conditions. Urothelial bladder carcinoma (BCa) cell lines T24 and TCCSUP were purchased from the American Type Culture Collection (ATCC ${ }^{\circledR}$, Manassas, VA) and were grown in a $5 \%$ $\mathrm{CO}_{2}$ incubator at $37^{\circ} \mathrm{C}$ and $85 \%$ humidity. Monolayer cultures were maintained in RPMI1640 medium (Biochrom), supplemented with $10 \%$ heat-inactivated fetal calf serum (FCS, Sigma, St. Louis, MO, USA), $1 \%$ streptomycin-penicillin antibiotics $\left(\mathrm{Gibco}^{\circledR}\right)$, and $1 \%$ glutamine (Thermo-Scientific Fisher, Darmstadt, Germany).

SiRNA-mediated MED30 knockdown. MED30 siRNA (sc-77529, Santa Cruz, TX, USA) was a pool of three target-specific siRNAs designed to knock-down gene expression. A non-targeting scrambled siRNA was used as the negative control (Exiqon Life Sciences, Copenhagen, Denmark). T24 and TCCSUP cells were transfected with $100 \mathrm{nmol} / \mathrm{L}$ siRNA using Screenfect A (Genaxxon Bioscience GmbH, Ulm, Germany) for 48 and $72 \mathrm{~h}$, respectively. Western blot. Post-transfected cells were washed with ice-cold phosphate buffered saline (PBS) and lysed in a protein extraction buffer for $30 \mathrm{~min}$. In addition, samples were sonicated for 2 minutes and subsequently centrifuged at $16,000 \times g$ for $30 \mathrm{~min}$ at $4^{\circ} \mathrm{C}$. Protein concentration was measured using bicinchoninic acid (BCA) Protein Assay Kit (Thermo Fisher Scientific), fractionated by SDSPage, and transferred into a nitrocellulose membrane. After incubation with $5 \%$ non-fat milk in TBST $(50 \mathrm{mM}$ Tris, $150 \mathrm{mM}$
$\mathrm{NaCl}, 0.05 \%$ Tween $20, \mathrm{pH} 7.5$ ) for $60 \mathrm{~min}$, the membrane was incubated with anti-MED30 rabbit polyclonal (1:200, clone 167871-AP, Proteintech, Chicago, IL, USA) or anti-GAPDH rabbit monoclonal $(1: 2,000$, clone 14C10, Cell Signaling Technology, Danvers, MA, USA) primary antibodies at $4^{\circ} \mathrm{C}$ overnight. Then, the membrane was washed three times for $5 \mathrm{~min}$ with TBST and incubated with horseradish peroxidase conjugated anti-rabbit antibody for $1 \mathrm{~h}$ at room temperature. Staining was detected by using SuperSignal West Femto Maximum Sensitivity Substrate, an ultra-sensitive enhanced chemiluminescent (ECL) substrat (Thermo Fisher Scientific).

Quantitative reverse transcription PCR ( $q R T-P C R)$. RNA was isolated from cell line pellets using the Total RNA Purification Mini Spin Column Kit (Genaxxon Bioscience GmbH). RNA quantity and quality was analysed using a NanoDrop 2000 spectrophotometer (Thermo Scientific). The cDNA was synthesized using $200 \mathrm{ng}$ total RNA and the PrimeScript RT Reagent Kit with gDNA Eraser (Takara Bio, Saint-Germain-en-Laye, France). Quantitative real-time PCR was performed by using $5 \mathrm{ng} / \mu \mathrm{l} \mathrm{cDNA}$, Takara Bio SYBR Premix Ex Taq II with ROX Plus and $10 \mathrm{pmol} / \mu \mathrm{l}$ forward and reverse primer. The following primer sequences were used: MED30 (Forward: 5'-ACCGGTTAACAAAGCTACAGGA-3', Reverse 5'TAAGTTGCTCGACTGGAATGGG-3'), and $\beta$-Actin (Forward 5'- 
CCAACCGCGAGAAGATGA-3'; Reverse 5'-CCAGAGGCGTA CAGGGATAG-3'). PCR was performed on an ABIPrism 7900 HT Fast Real-Time PCR System (Applied Biosystems, Foster City, CA, USA). Data were analyzed by using Qbase+ (Biogazelle, Ghent, Belgium) with $\beta$-Actin (ACTB) as the reference gene, applying the 2- $\Delta \Delta \mathrm{CT}$ algorithm.

EZ4U cell proliferation assay. The EZ4U cell proliferation assay kit was used according to the manufacturer's protocol (EZ4U; Biomedica Group, Vienna, Austria). The siRNA transfections for proliferation assays were performed for 96-well plates. In each well of a flat-bottom 96 -well plate, either $1.2 \times 10^{4}$ cells (T24) or $2.0 \times 10^{4}$ cells (TCCSUP) were seeded in $200 \mu$ cell culture medium. SiRNA mediated MED30 knockdown was then performed and cells were incubated to adhere and grow for $72 \mathrm{~h}$. After incubation, $20 \mu \mathrm{l}$ of EZ4U substrate solutions were added before incubating for $3 \mathrm{~h}$ (T24) and $4 \mathrm{~h}$ (TCCSUP), until the colour of the solution changed from yellow to orange. The absorbance was measured by using a microplate reader (Tecan, Model Spectra Thermo) at $450 \mathrm{~nm}$ wavelength. Each experiment was repeated at least three times.

Migration assays. The siRNA transfections for migration assays were performed in 6-well plates. 48hours post-transfection, cells were trypsinized and seeded into migration boyden chambers. $3 \times 10^{4}$ cells (T24) or $5 \times 10^{4}$ cells (TCCSUP) were plated in the upper chamber of migration inserts (VWR, Darmstadt, Germany) containing $0 \%$ FCS medium. The lower chamber was filled with a medium containing $10 \%$ FCS for chemotactic attraction. After $24 \mathrm{~h}, \mathrm{BCa}$ cells were fixed with $4 \%$ paraformaldehyde (Merck, Darmstadt, Germany), stained with haematoxylin (Waldeck, Münster, Germany), and washed with water. Membranes were scanned and manually evaluated by counting. Each experiment was repeated at least three times.

Invasion assays. The siRNA transfections for invasion assays were performed in 6-well plates. $48 \mathrm{~h}$ post-transfection, cells were trypsinized and seeded into matrigel invasion chambers. $7.5 \times 10^{4}$ cells were plated in the upper chamber of invasion inserts (VWR, Darmstadt, Germany), containing 0\% FCS medium. The lower chamber was filled with a medium containing 10\% FCS for chemotactic attraction. After $24 \mathrm{~h}, \mathrm{BCa}$ cells were fixed with $4 \%$ paraformaldehyde (Merck, Darmstadt, Germany), stained with haematoxylin (Waldeck, Münster, Germany), and washed with water. Membranes were scanned and manually evaluated through counting. Each experiment was repeated at least three times.

\section{Results}

Transcriptional expression of the MED30 by cBioPortal. Using the cBioPortal database, we analyzed the mRNA expression of MED30 in bladder urothelial carcinoma samples. In two analyses, 129 and 408 samples of bladder urothelial carcinoma (given by TCGA) were investigated. The results of the expression analyses are shown in Figures 2 and 3 and those for overall and disease-free survival in Figure 4.

Transcriptional $m R N A$ expression regarding the database cBioPortal. In the first selected analysis, named "Bladder Urothelial Carcinoma TCGA, Provisional", an alteration of
MED30 is described in $20 \%(80 / 408)$ of the samples. In detail, 79 up-regulations and one down-regulation of the mRNA expression of MED30 were found (Figure 2).

The second analysis, called "Bladder Urothelial Carcinoma TCGA, Nature 2014", included 129 samples. An alteration of MED30 is described in 20\% (26/129) of the samples. In detail, 25 samples showed up-regulation, and one sample showed down-regulation of MED30 mRNA expression (Figure 2).

As shown in Figure 3, the alterations of MED30 in BCa are especially gain-of-function-mutations and amplifications. Deletions are rare.

Survival analysis regarding transcriptional expression. In the analysis, "Bladder Urothelial Carcinoma TCGA Provisional", the overall survival divided by an altered MED30 mRNA expression showed a tendency, unfortunately not significant, for samples with an alteration of MED30 (predominantly overexpression) to have better overall survival (log rank test: $p=0.361$; samples with alteration (97.04 months) $v s$. without alteration (33.11 median survival months) (Figure 4A).

Considering disease-free survival, the results showed the same nonsignificant tendency ( $\log$ rank test: $p=0.708$; samples with alteration (55.16 months) vs. without alteration (30.12 median disease-free months) (Figure 4B).

In the analysis called "Bladder Urothelial Carcinoma TCGA, Nature 2014", the overall survival of samples with alteration of $M E D 30$ (predominantly overexpression) showed a significantly better overall survival than patients without alteration [ $\log$ rank test: $p=0.01$; with alteration (N.A. $=$ not available) $v s$. without alteration (median 19.49 months' survival)] (Figure 4C).

Contradictory results are given in this analysis for diseasefree survival. For this, samples with alteration performed worse than those without alteration of $M E D 30$ mRNA expression, but the results were not significant (log rank test: $p=0.541 ; 6.93$ vs. 8.54 median disease-free months (Figure 4D).

In conclusion, in the analysis of the database cBioPortal, an approximately $20 \%$ up-regulation of the mRNA level was found with an influence on the overall expression. For overall survival, a clear tendency towards a significant difference $(p=0.01)$ existed for the groups with and without alterations. Interestingly, patients with amplified and overexpressed $M E D 30$ had a favourable outcome, suggesting MED30 amplification as a predictive marker.

Immunohistochemistry. To validate the transcriptional mRNA data obtained from the analysis of the cBioPortal database, we performed clinical analyses on large tissue microarray (TMA) cohorts with the available clinical information.

For the calculation of MED30 protein expression in the progression of urothelial bladder cancer, a large tissue microarray (TMA) including $210 \mathrm{BCa}$ patient samples (Table I) of all tumour stages and benign urothelium was analysed by immunohistochemistry (IHC) followed by IRS analysis. 
A

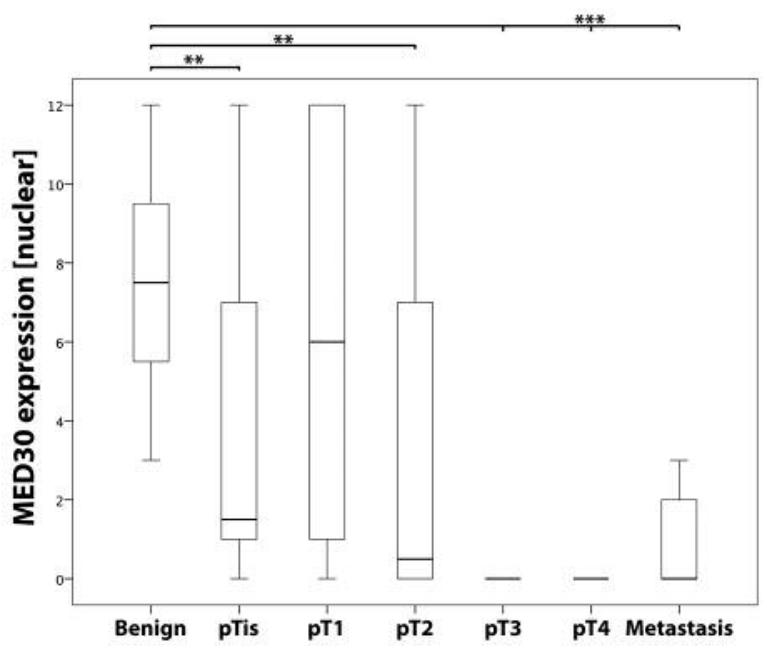

C

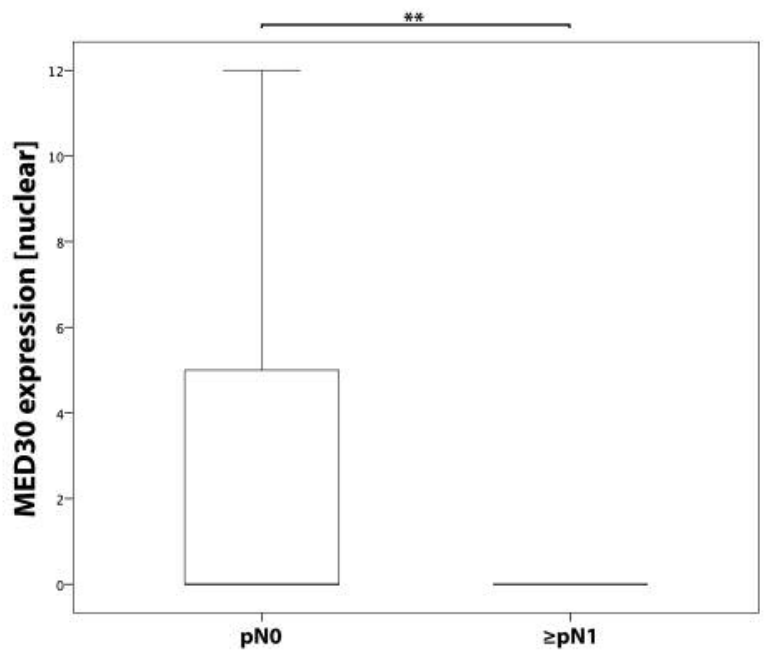

B

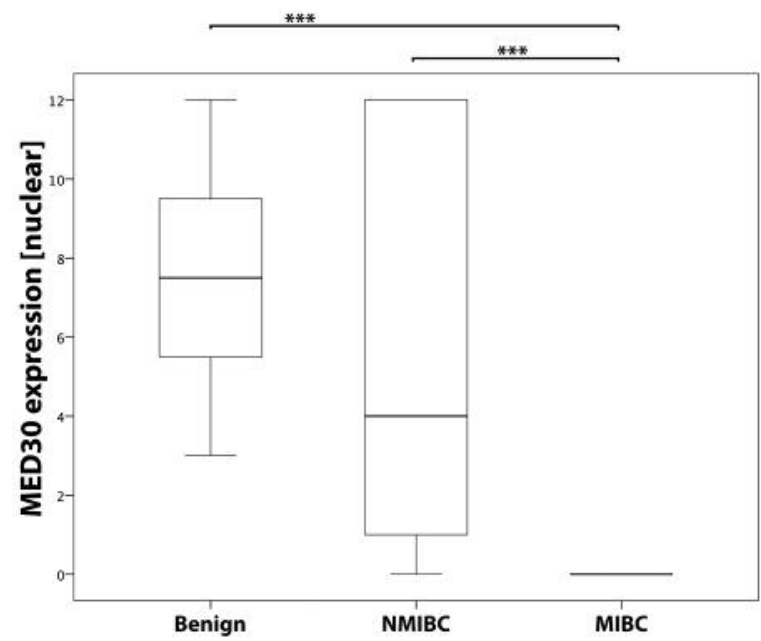

D

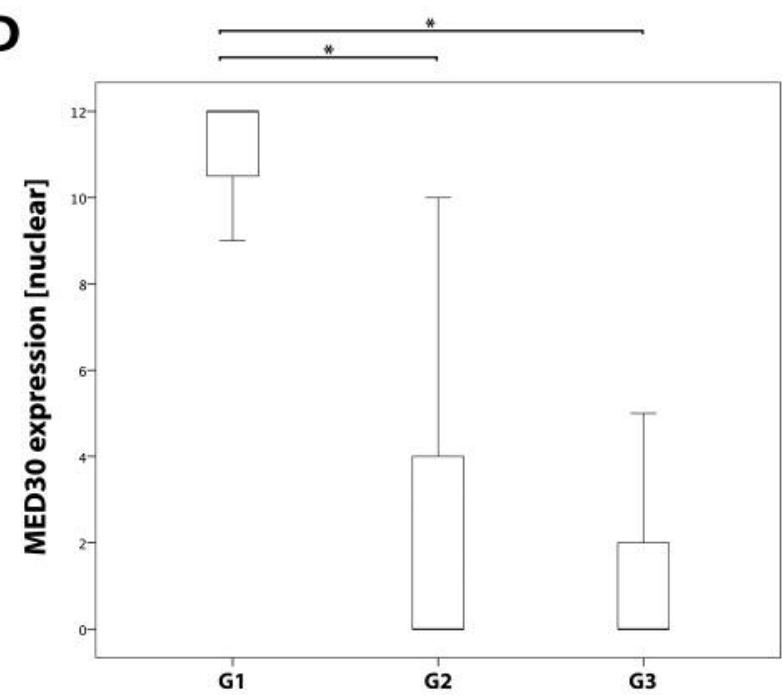

Figure 6. IHC analysis for MED30 in BCa. (A) Mean IRS score for MED30 protein expression profile of the total bladder cohort including benign urothelium, pTis, T1, T2, T3, T4, and metastasis BCa tissue. (B) The average nuclear MED30 staining intensity (IRS) of tissue samples decreases significantly throughout disease stages. (C) The simultaneous presence of lymph node metastases is significantly associated with a lower MED30 expression. (D) Higher grading of the tumour is significantly associated with lower MED30 expression. Bars and error bars indicate mean IRS $\pm S D$. Independent-samples $t$-test, ${ }^{*} p \leq 0.05, * * p \leq 0.01, * * * p \leq 0.001$.

In the immunohistochemical analysis, we investigated the expression of MED30 in the cell nuclei. Cytoplasmic expression was very rare and seemed not to be specific (Figure 5). The highest nuclear expression of MED30 was found in normal tissue (median IRS=7.5, SD 2.918). Regarding progression, higher tumour stages were associated with a significantly lower expression of MED30 (T1 IRS $=6.00$, SD 5.105, $p=0.767$, up to T4 IRS $=0.00$, SD $0.177, p \leq 0.001$, and metastases IRS $=0.00$, SD 1.820 , $p \leq 0.001$ ) (Figures 5 and 6A). Further, in non-muscle invasive $\mathrm{BCa}(\mathrm{NMIBC}=\mathrm{pTis}$ and $\mathrm{pT} 1$; IRS $=4.00, \mathrm{SD} 0.177$ ) the expression intensity of MED30 was lower compared with the benign transitional epithelium (Figure 6B, $p=0.228$ ) and further falling in muscle-invasive tumour stages $(\mathrm{MIBC}=\mathrm{T} 2$ T4; IRS $=0.00$, SD 1.820, $p \leq 0.001$ ) (Figure 6B).

The expression of MED30 in pTis was lower (IRS=1.5, SD 4.266, $p=0.094)$ than the expression in control tissue (IRS=7.50, SD 2.918) or pT1 (IRS=6.00, SD 5.105) (Figure $6 \mathrm{~A})$, which may also underline the clinical relevance of Tis, which is associated with a significantly higher risk of tumour progression. Overall, the expression of MED30 decreased significantly from benign tissue over NMIBC up to MIBC. Furthermore, positive lymph node status $(\geq \mathrm{N} 1, \mathrm{IRS}=0.00$, $\mathrm{SD} 3.509, p=0.005$, Figure $6 \mathrm{C}$ ) and advanced grading (G3; 

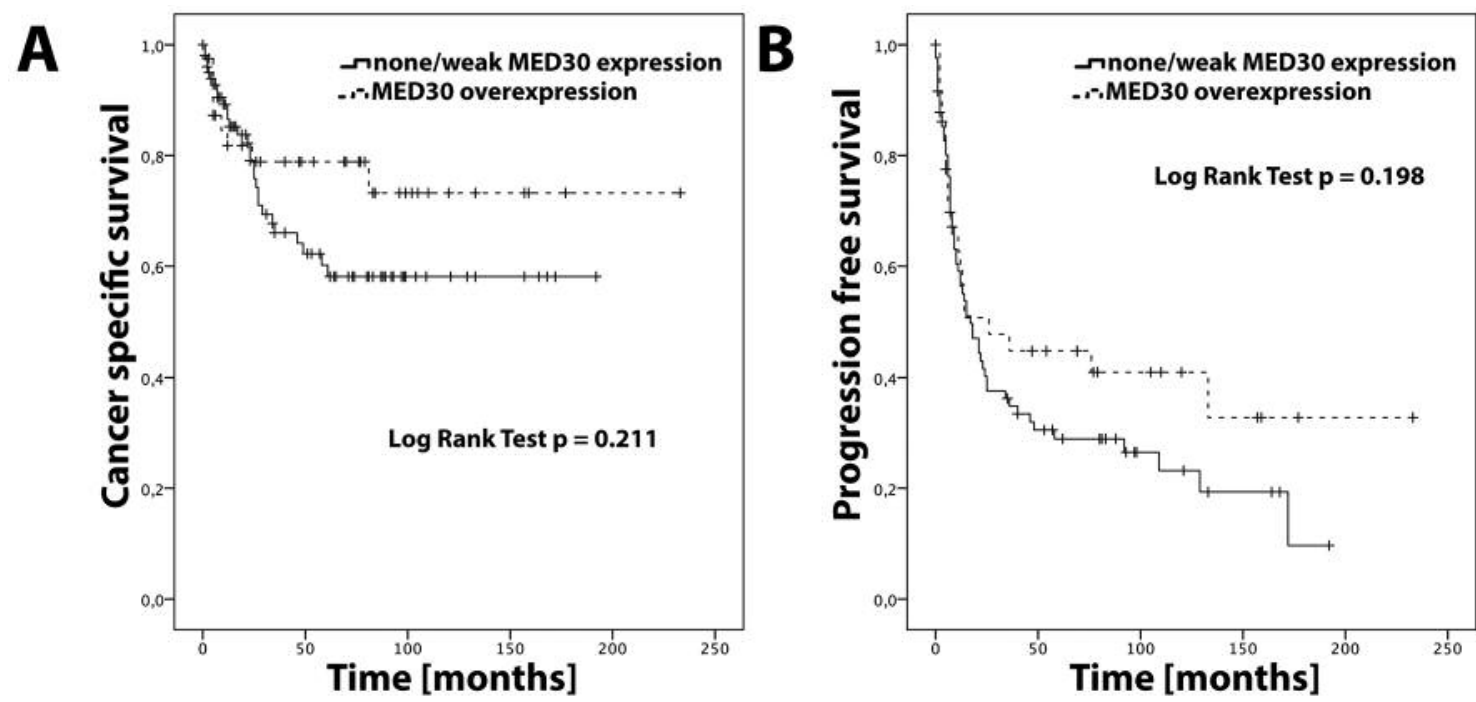

Figure 7. Survival analysis for MED30 in the IHC-analysis. Kaplan-Meier analysis for cancer-specific survival (A) and progression-free survival (B) according to the expression of MED30. An IRS $\geq 4$ was defined as an overexpression of MED30. Samples without overexpression of MED30 show a non-significant tendency toward longer survival.

IRS $=0.00, \mathrm{SD} 4.080, p=0.016$, Figure 6D) were associated with lower MED30 expression. In contrast to this and to the lowest expression being found in metastases, a positive metastatic status (M1) was not associated with lower MED30 expression ( $p=0.78$, data not shown), probably due to the low numbers of metastasized samples $(n=39$, Table I). Additionally, the expression of MED30 was significantly lower in metastases ( $p \leq 0.001$ Figure 6A).

According to these results, patients with low MED30 protein expression showed a tendency towards an unfavourable clinical outcome and decreased survival evaluated by Kaplan-Meier estimator and log-rank test (Figure 7). Unfortunately, this relationship was not significant (cancer-specific survival $p=0.211$, progressionfree survival $p=0.198$ ).

Functional analysis. After the knockdown of the subunit MED30 mediated by siRNA, the results of functional analysis are in complete contrast expected according to the results given by of cBioportal and the IHC.

Shown in Figure 8, the siRNA mediated knockdown led to a significant reduction of the proliferation (T24 $p \leq 0.001$, TCCSUP $p \leq 0.001$ ), migration (T24 $p=0.002$, TCCSUP $p \leq 0.001$ ), and invasion (T24 $p \leq 0.001$, TCCSUP $p \leq 0.001$ ).

In conclusion, MED30 protein expression decreased during the progression from benign to high tumour stages and seems to be play a tumour supressing role on protein level. The contrasting role was found in the functional analysis in the cell lines.

\section{Discussion}

Since the discovery of the Mediator complex by Kornberg in yeast and humans (20), multiple investigations in to different Mediator complex subunits in diseases such as cancer have been performed, and altered expression of the subunits have been found. The mediator complex is an important regulator of the transcription machinery and consists of $\sim 33$ subunits. The head and middle module interact with the polymerase II, whereas the tail and kinase module communicate with transcription factors and link up to signalling pathways (47). To date, the role of the subunit MED30 in the progression of bladder urothelial carcinoma has never been investigated.

MED30 is part of the tail module and altered expressions of $M E D 30$ has been described in several diseases, although the exact functional role is only poorly understood. In HIV, the knockdown of the $M E D 30$ proteins significantly impaired the viral replication (21). Alternative transcripts of $M E D 30$ subunit expressed only in circulating immature progenitor cells (22). For cardiomyopathy, a missense mutation in MED30 causing progressive cardiomyopathy in homozygous mice (23). Various breast carcinoma cell lines expressed abundant amounts of MED1, MED24, and MED30 (8). Furthermore, a MED30 overexpression was found in gastric cancer cells (9).

In our study, we examined the role of MED30 in urothelial cancer. In our first investigation, we utilized the platform cBioPortal and found out that an overexpression of MED30 on the mRNA level is associated with a tendency toward 


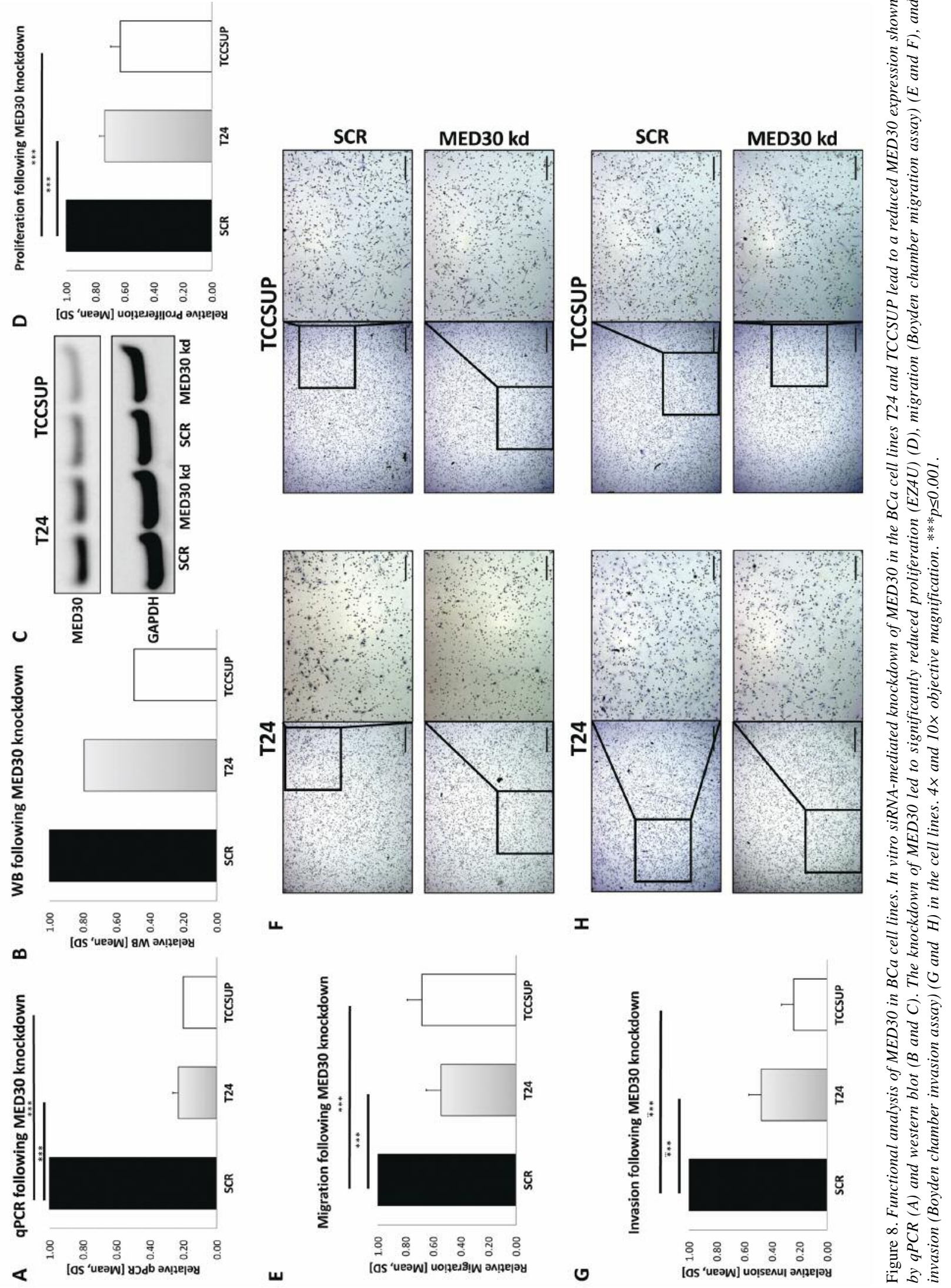


favourable patient survival (Figure 4). Our results of the immunohistochemistry supported these findings: higher tumour stages, like T3 and T4 (MIBC) (Figure 6A and B), the presence of lymph node metastases (Figure 6C) and higher grading of the tumour (Figure 6D), which are associated with a worse outcome, showed a strongly reduced expression of MED30. This leads us to the conclusion that MED30 appears to have a tumour-suppressing role in bladder cancer. However, the results of the functional analysis diverge completely to the results of the cBioportal and the IHC: the knockdown of MED30 reduced the proliferation, migration, and invasion of the BCa cell lines. These parameters are crucial for the progression of tumours and necessary for the metastatic spread. The reduction of theses parameters after the knockdown suggest an oncogenic impact of MED30 in bladder carcinoma.

The role as a tumour suppressor has already been described for other subunits such as MED1 in melanoma (24), lung cancer $(25,26)$ and urothelial cancer $(27)$. In melanoma, the down-regulation of the tail module $M E D l$ triggers a strong tumorigenic phenotype (24) and is associated with worse outcome in lung adenocarcinoma (25). Interestingly, loss of MED1 promotes the appearance of metastases of non-smallcell lung cancer cells by modulating metastasis-related genes (26) and was associated with the down-regulation of tumour suppressor gene dapkl (28).

Additionally, it must be discussed that other mediator subunits in different cancer entities have an oncogenic, not a suppressing, character. The reason for this can be found in the connection to different signalling pathways (like Wnt- or TGF $\beta$-signalling pathway) or to further transcription factors (coactivators $v s$. inhibitors). Additionally, a possible explanation might be that some subunits, such as MED1, as coactivators of nuclear hormone receptors, are necessary in hormone-dependent tumours such as prostate (29) or breast cancer (30), whereas in other cancer entities such as melanoma (24), lung cancer (25) and BCa (27), downregulation of $M E D 1$ increases tumorigenic potential by modulating metastasis-related genes such as uPAR.

Differences in activating or inhibiting character can even be found in the same subunit. In another of our analyses, we investigated MED30 in renal carcinoma (manuscripct in preparation). These results showed a partly deviating tendency towards the results for bladder cancer: in the progression to higher tumour stages, the expression of MED30 increased in the IHC analysis. This might be explained by the completely different origins of the tumours and gene profiles involved. For example, in bladder cancer, $\mathrm{N}$-acetyltransferases are of special interest, the so-called "ultra-slow" acetylators have a higher risk for bladder cancer $(31,32)$. Additionally, enhanced expression of oncogenes such as ras and C-MYC or reduced activity of tumour suppressor genes such as p53, p21 or retinoblastoma-gen $\mathrm{Rb}$ are frequent in bladder cancer (33). In contrast, for renal cell carcinoma, overexpression of VEGF/R-, PDGF, TGF and FGF induced by hypoxia is known $(34,35)$.

The divergent characters of the same subunit in the same tumour entity have not been described yet and require further investigations and validation for a possible explanation.

\section{Conclusion}

MED30 appears to be integrated in the progression of urothelial bladder cancer. Further investigations are necessary to discover which signaling pathways and regulatory procedures are the important steps in the progression of bladder cancer.

\section{Conflicts of Interest}

The Authors declare no conflicts of interest.

\section{Acknowledgements}

The study was supported by the Ferdinand Eisenberger-Fellowship of the German Society of Urology (DGU) (SYI1/FE-13), and the Maria von Linden-Program of the University of Bonn for IS.

\section{References}

1 Robert Koch Institut, Krebs in Deutschland 2011/2012 10. Ausgabe, 2015. Zentrum für Krebsregisterdaten Gesundheitsberichterstattung des Bundes.

2 Wang H, Yuan G, Zhao B, Zhao Y and Qiu Y: High expression of $\mathrm{B} 23$ is associated with tumorigenesis and poor prognosis in bladder urothelial carcinoma. Mol Med Rep 15(2): 743-749, 2017.

$3 \mathrm{Wu}$ TF, Wu H, Chow NH, Liao CF and Liu HS: Proteomic analysis of bladder cancer cells reveals potential candidates of biomarkers in bladder tumorigenesis. Cancer Genomics Proteomics 2: 151-158, 2005.

4 Ansari SA and Morse RH: Mechanisms of Mediator complex action in transcriptional activation. Cell Mol Life Sci 70(15): 2743-2756, 2013.

5 Casamassimi A and Napoli C: MED complexes and eukaryotic transcription regulation: an overview. Biochimie 89(12): 14391446, 2007.

6 Boube M, Joulia L, Cribbs DL and Bourbon HM: Evidence for a MED of RNA polymerase II transcriptional regulation conserved from yeast to man. Cell 110(2): 143-151, 2002.

7 Napoli C, Sessa M, Infante T and Casamassimi A: Unraveling framework of the ancestral MED complex in human diseases. Biochimie 94(3): 579-587, 2012.

8 Hasegawa N, Sumitomo A, Fujita A, Aritome N, Mizuta S, Matsui K, Ishino R, Inoue K, Urahama N, Nose J, Mukohara T, Kamoshida S, Roeder RG and Ito M: Mediator subunits MED1 and MED24 cooperatively contribute to pubertal mammary gland development and growth of breast carcinoma cells. Mol Cell Biol 32(8): 1483-1495, 2012.

9 Lee YJ, Han ME, Baek SJ, Kim SY and Oh SO: MED30 regulates the proliferation and motility of gastric cancer cells. PLoS One 10(6): eCollection, 2015. 
10 Gao J, Aksoy BA, Dogrusoz U, Dresdner G, Gross B, Sumer SO, Sun Y, Jacobsen A, Sinha R, Larsson E, Cerami E, Sander $\mathrm{C}$ and Schultz $\mathrm{N}$ : Integrative analysis of complex cancer genomics and clinical profiles using the cBioPortal. Sci Signal 6(269), 2013.

11 Cerami E, Gao J, Dogrusoz U, Gross BE, Sumer SO, Aksoy BA, Jacobsen A, Byrne CJ, Heuer ML, Larsson E, Antipin Y, Reva B, Goldberg AP, Sander C and Schultz N: The cBio cancer genomics portal: an open platform for exploring multidimensional cancer genomics data. Cancer Discov 2(5): 401-404, 2012.

12 Tomczak K, Czerwińska P and Wiznerowicz M: The Cancer Genome Atlas (TCGA): an immeasurable source of knowledge. Contemp Oncol (Poznan, Poland) 19(1A): A68-77, 2015.

13 Scheble VJ, Braun M, Wilbertz T, Stiedl AC, Petersen K, Schilling D, Reischl M, Seitz G, Fend F, Kristiansen G and Perner S: ERG rearrangement in small cell prostatic and lung cancer. Histopathology 56(7): 937-943, 2010.

14 Scheble VJ, Braun M, Beroukhim R, Mermel CH, Ruiz C, Wilbertz T, Stiedl AC, Petersen K, Reischl M, Kuefer R, Schilling D, Fend F, Kristiansen G, Meyerson M, Rubin MA Bubendorf $\mathrm{L}$ and Perner S: ERG rearrangement is specific to prostate cancer and does not occur in any other common tumor. Mod Pathol 23(8): 1061-1067, 2010.

15 Syring I, Klümper N, Offermann A, Braun M, Deng M, Boehm D, Queisser A, von Mässenhausen A, Brägelmann J, Vogel W, Schmidt D, Majores M, Schindler A, Kristiansen G, Müller SC, Ellinger J, Shaikhibrahim Z and Perner S: Comprehensive analysis of the transcriptional profile of the Mediator complex across human cancer types. Oncotarget 7(17): 23043-23055, 2016.

16 Brägelmann J, Klümper N, Offermann A, von Mässenhausen A, Böhm D, Deng M, Queisser A, Sanders C, Syring I, Merseburger AS, Vogel W, Sievers E, Vlasic I, Carlsson J, Andrén O, Brossart P, Duensing S, Svensson MA, Shaikhibrahim Z, Kirfel J and Perner $S$ : Pan-cancer analysis of the Mediator complex transcriptome identifies CDK19 and CDK8 as therapeutic targets in advanced prostate cancer. Clin Cancer Res [Epub ahead of print], 2016.

17 Klümper N, Syring I, Offermann A, Shaikhibrahim Z, Vogel W, Müller SC, Ellinger J, Strau $\beta$ A, Radzun HJ, Ströbel P, Brägelmann J, Perner $S$ and Bremmer F: Differential expression of Mediator complex subunit MED15 in testicular germ cell tumors. Diagn Pathol 10: 165, 2015.

18 Braun M, Scheble VJ, Menon R, Scharf G, Wilbertz T, Petersen K, Beschorner C, Reischl M, Kuefer R, Schilling D, Stenzl A, Kristiansen G, Rubin MA, Fend F and Perner S: Relevance of cohort design for studying the frequency of the ERG rearrangement in prostate cancer. Histopathology 58(7): 1028-1036, 2011.

19 Remmele W and Stegner HE: Recommendation for uniform definition of an immunoreactive score (IRS) for immunohistochemical estrogen receptor detection (ER-ICA) in breast cancer tissue. Pathologe 8(3): 138-140, 1987.

20 Myers LC and Kornberg RD: Mediator of transcriptional regulation. Annu Rev Biochem 69: 729-749, 2000.

21 Ruiz A, Pauls E, Badia R, Riveira-Muñoz E, Clotet B, Ballana $\mathrm{E}$ and Esté JA: Characterization of the influence of mediator complex in HIV-1 transcription. J Biol Chem 289(40): 2766527676, 2014.

22 Rienzo M, Casamassimi A, Schiano C, Grimaldi V, Infante T and Napoli C: Distinct alternative splicing patterns of mediator subunit genes during endothelial progenitor cell differentiation. Biochimie 94(8): 1828-1832, 2012.
23 Krebs P Fan W, Chen YH, Tobita K, Downes MR, Wood MR, Sun L, Li X, Xia Y, Ding N, Spaeth JM, Moresco EM, Boyer TG, Lo CW, Yen J, Evans RM and Beutler B: Lethal mitochondrial cardiomyopathy in a hypomorphic Med30 mouse mutant is ameliorated by ketogenic diet. Proc Natl Acad Sci USA 108(49): 19678-19682, 2011.

24 Ndong J de L, Jean D, Rousselet $\mathrm{N}$ and Frade R: Downregulation of the expression of RB18A/MED1, a cofactor of transcription, triggers strong tumorigenic phenotype of human melanoma cells. Int J Cancer 124(11): 2597-2606, 2009.

25 Yun J, Son CH, Um SJ, Kwon HC, Lee KE, Choi PJ and Roh MS: A different TRAP220 expression in distinct histologic subtypes of lung adenocarcinoma and the prognostic significance. Lung Cancer 71(3): 312-318, 2011.

26 Kim HJ, Roh MS, Son CH, Kim AJ, Jee HJ, Song N, Kim M, Seo SY, Yoo YH and Yun J: Loss of Med1/TRAP220 promotes the invasion and metastasis of human non-small-cell lung cancer cells by modulating the expression of metastasis-related genes. Cancer Lett 321(2): 195-202, 2012.

27 Klümper N, Syring I, Vogel W, Schmidt D, Müller SC, Ellinger $\mathrm{J}$, Shaikhibrahim Z, Brägelmann $\mathrm{J}$ and Perner S: Mediator complex subunit MED1 protein expression is decreased during bladder cancer progression. Front Med 4(30): 1-6, 2017

28 Gade P, Singh AK, Roy SK, Reddy SP and Kalvakolanu DV: Down-regulation of the transcriptional mediator subunit Med1 contributes to the loss of expression of metastasis-associated dapk1 in human cancers and cancer cells. Int J Cancer 125(7): 1566-1574, 2009.

29 Vijayvargia R, May MS and Fondell JD: A coregulatory role for the Mediator complex in prostate cancer cell proliferation and gene expression. Cancer Res 67(9): 4034-4041, 2007.

30 Cui J, Germer K, Wu T, Wang J, Luo J, Wang SC, Wang Q and Zhang X: Cross-talk between HER2 and MED1 regulates tamoxifen resistance of human breast cancer cells. Cancer Res 72(21): 5625-5634, 2012.

31 Selinski S, Blaszkewicz M, Ickstadt K, Hengstler JG and Golka $\mathrm{K}$ : Refinement of the prediction of $\mathrm{N}$-acetyltransferase 2 (NAT2) phenotypes with respect to enzyme activity and urinary bladder cancer risk. Arch Toxicol 87(12): 2129-2139, 2013.

32 Lin SS, Yu CS, Wu JY, Tyan YS, Hsia TC, Lin WC and Chung JG: Effects of Aspirin on the In Vitro and In Vivo Acetylation of 2-Aminofluorene in Sprague-Dawley Rats. In Vivo 19(2): 475-481, 2005.

33 Baffa R, Letko J, McClung C, LeNoir J, Vecchione A and Gomella LG: Molecular genetics of bladder cancer: targets for diagnosis and therapy. J Exp Clin Cancer Res 25(2): 145-160, 2006.

34 Duran I, Lambea J, Maroto P, González-Larriba JL, Flores L, Granados-Principal S, Graupera M, Sáez B, Vivancos A and Casanovas O: Resistance to targeted therapies in renal cancer: The importance of changing the mechanism of action. Target Oncol 12(1): 19-35, 2017.

35 Sherbet GV: Metastasis promoter S100A4 is a potential molecular therapeutic target. Cancer Genomics Proteomics 3(34): 203-216, 2006.

Received August 30, 2017

Revised October 4, 2017

Accepted October 11, 2017 